\title{
Therapeutic management in a child with Wilms tumour and radiological features suggesting rupture at the time of diagnosis
}

\author{
Patrycja Sosnowska-Sienkiewicz ${ }^{1}$, Danuta Januszkiewicz-Lewandowska², \\ Katarzyna Jończyk-Potoczna ${ }^{3}$, Ewelina Gowin ${ }^{4}$, Przemysław Mańkowski ${ }^{1}$ \\ 'Department of Paediatric Surgery, Traumatology and Urology, Poznan University of Medical Sciences, Poznan, Poland \\ 2Department of Paediatric Oncology, Haematology and Transplantology, Poznan University of Medical Sciences, Poznan, Poland \\ ${ }^{3}$ Department of Paediatric Radiology, Poznan University of Medical Sciences, Poznan, Poland \\ ${ }^{4}$ Department of Health Promotion, Poznan University of Medical Sciences, Poznan, Poland
}

\section{ABSTRACT}

Preoperative Wilms tumour rupture has been reported sporadically. But when identified, SIOP (Societe Internationale d'Oncologie Pediatrique) recommends that these patients should be upstaged to the $3^{\text {rd }}$ stage. The purpose of this publication was to present the clinical and radiological features of preoperative tumour rupture in our patient.

A 4-year-old girl was treated in the oncology department for a large Wilms tumour. The child presented inconclusive clinical and radiographical features of preoperative tumour rupture. Therefore, standard 4-week preoperative chemotherapy was used. Unfortunately, a tight connection between the cystic part of the tumour and the peritoneum became the cause of tumour rupture during surgery.

Not every child who meets the radiological criteria for preoperative tumour rupture should be upgraded. The radiological features of preoperative tumour rupture shouldvery much increase the caution of the surgeon.

KEY WORDS:

child, computed tomography, surgery, tumour rupture, Wilms tumour.

\section{INTRODUCTION}

Wilms tumour is the most common kidney cancer in children [1]. It is characterized by chemo- and radiosensitivity, otherwise its fragility is associated with a risk of tumour rupture [2]. The last one is a major risk factor for recurrence of the tumour. To reduce the likelihood of a tumour rupture during surgery, International Society of Paediatric Oncology (Societe Internationale d'Oncologie Pediatrique [SIOP]) recommends preoperative treatment of patients with neoadjuvant chemotherapy [3]. Pre-operative Wilms tumour rupture with anaemia, pain and acute abdomen is reported incidentally. But once identified during diagnosis, SIOP recommends that these patients be upstaged to the $3^{\text {rd }}$ stage, regardless of the outcome of the histopathology after surgery. This results in more intense chemo- and radiation therapy. Other ambiguous clinical and radiological features suggesting preoperative tumour rupture are described in the literature, but their role remains debatable and they do not clearly indicate a specific procedure [4].

The purpose of this publication was to present the clinical and radiological features of preoperative tumour rupture in our patient in relation to those described in the literature. We believe that such an analysis performed in every patient would be helpful for surgeon before removing the Wilms tumour.

\section{ADDRESS FOR CORRESPONDENCE:}

Patrycja Sosnowska-Sienkiewicz, Department of Paediatric Surgery, Traumatology and Urology, Poznan

University of Medical Sciences, Poznan, Poland, e-mail: patrycja.sosnowska@outlook.com 


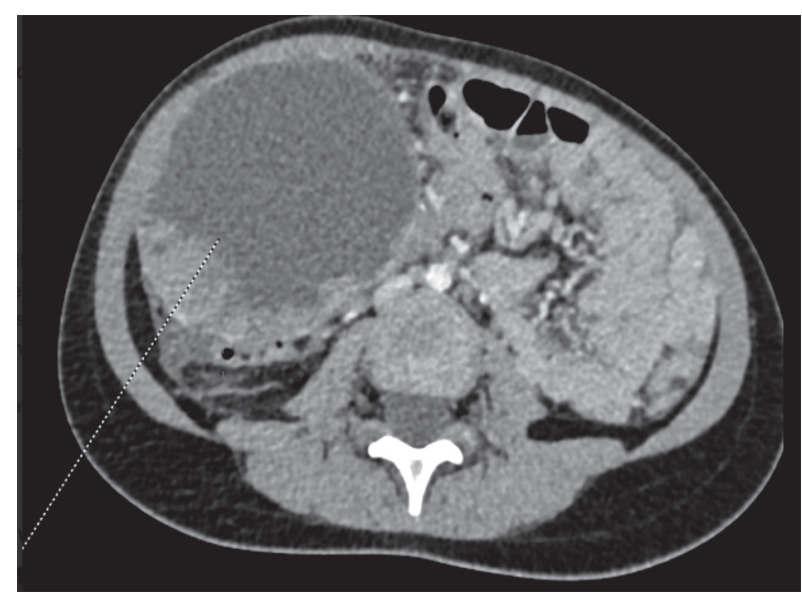

FIGURE 1. CT in transverse plane with right kidney tumor. The capsular break and retroperitoneal fluid in the subcapsular, perirenal, and pararenal space was observed

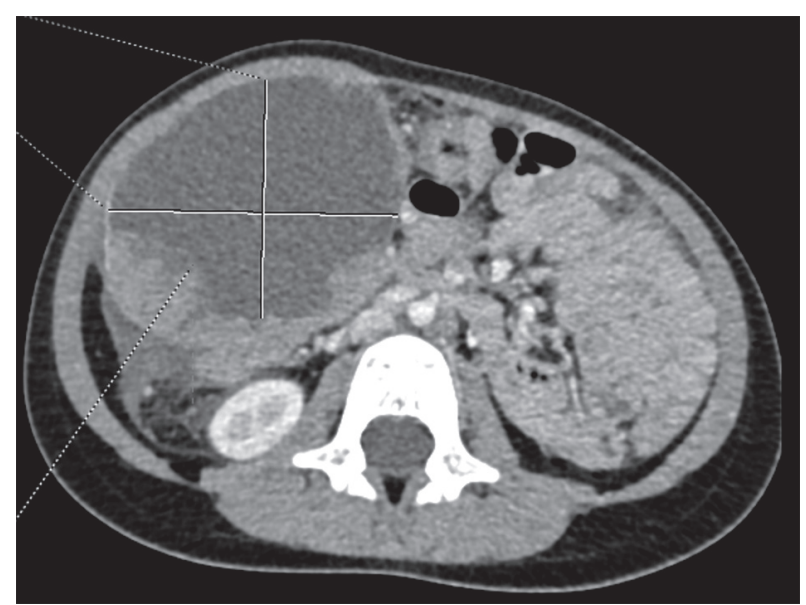

FIGURE 2. CT in transverse plane with right kidney tumor and fat stranding around tumor (linear areas of soft-tissue attenuation in the fat around the tumor)

TABLE 1. Complete blood count in our patient during admission to the hospital

\begin{tabular}{|l|c|c|}
\hline Parameter (unit) & Patient's result & Normal value \\
\hline Haemoglobin (g\%) & 9,5 & $10-15$ \\
\hline Haematocrit $(\%)$ & 27 & $30-45$ \\
\hline White blood cells $\left(10^{9} / \mathrm{l}\right)$ & 17 & $8-15$ \\
\hline Platelets $\left(10^{\circ} / \mathrm{l}\right)$ & 467 & $150-300$ \\
\hline
\end{tabular}

\section{CASE REPORT}

A 4-year-old girl was admitted to the oncology department because of an abdominal tumour. Two days earlier, sudden abdominal pain at night and a single fever incident were observed. No abdominal injury has been reported. At admission, the child was in a good condition, and was painless during palpation. Ultrasound, CT (computer tomography) and scintigraphy indicated

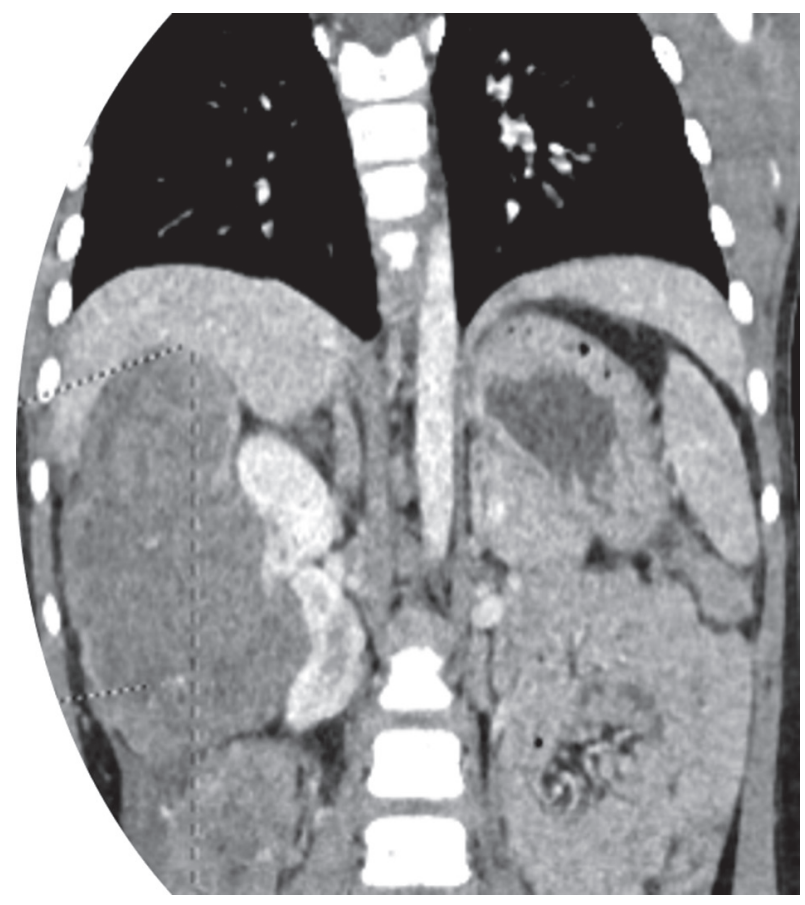

FIGURE 3. CT in frontal plane with poorly circumscribed tumor of right kidney and intraabdominal fluid

a large tumour with the cystic part of the right kidney, without metastases to the lungs and liver. The tumour was heterogeneous, hypodense; $9 \times 10 \times 16 \mathrm{~cm}$ in size. Calcification and a few hypodense liquid areas were visible; the largest was $8 \times 7 \times 10 \mathrm{~cm}$ in size. Additionally, in the lower pole of the kidney a hypodense lesion, $17 \times$ $18 \times 23 \mathrm{~mm}$ in size, with calcification was found (Figures 1, 2 and 3). Upwards, the tumour adhered to the visceral surface of the right lobe of the liver without maintaining peri-organ fat reserve. This suggested infiltration. A small amount of free retroperitoneal fluid in the subcapsular, perirenal, and pararenal space was visible (Figures 1 and 3). A capsular break and numerous, enlarged lymph nodes were found.

Laboratory tests showed anaemia and a slightly higher number of leukocytes and platelets (Table 1).

The child was given pre-operative 4-weeks' chemotherapy (vincristine and actinomycin according to SIOP WILMS 2001). CT performed after this treatment showed that the tumour dimensions did not change. This was mainly due to the presence of intra-tumour cysts that remained as large as in the first CT scan. A decision was made to remove the tumour. A transperitoneal approach was used. During the surgery a tumour closely related to the peritoneum was found. This caused a rupture of a cystic part of the tumour. Radical right nephrectomy was performed with lymph node sampling. Apart from the described tumour rupture, no other complications occurred. In the histopathological examination the nephroblastoma-diffuse anaplasia type was found. A high-risk Wilms tumour ( $3^{\text {rd }}$ stage acc. to SIOP WILMS 2001) with an infiltration of liver and peritoneum was diagnosed. 
This child needed an aggressive treatment and whole abdominal irradiation acc. to the protocol. The child has finished treatment and has been in a complete remission of the disease for two years.

\section{DISCUSSION}

Pre-operative tumour rupture is a significant risk factor for recurrence the Wilms tumour $[5,6]$. Wilms tumour grow quickly and are often very large when a child is admitted to hospital. This makes the tumour prone to spontaneous rupture, or it can be after an unnoticed trauma. In Europe, the diagnosis of preoperative rupture is based on the history, imaging, surgery and histopathology after pre-operative chemotherapy [7]. However, abdominal CT is not always conclusive in detecting pre-operative Wilms tumour rupture. Moreover, radiological markers of tumour rupture are also not always confirmed by surgery and histopathological examination [8].

Le Rouzic et al. performed a retrospective analysis of patients treated for Wilms tumour [2]. The clinical criteria for tumour rupture included: intense abdominal pain, history of recent abdominal trauma and acute haemorrhage with rapid decrease in haemoglobin level. Radiological features for tumour rupture included: capsular rupture, haemorrhage inside the tumour or in the perirenal space, retroperitoneal and/or intraabdominal effusion. The analysis was based on CT and histopathological results. Clinical manifestations of tumour rupture were observed in 22 out of 28 patients and radiological symptoms in up to 24 children. Histopathological studies confirmed the rupture only in 5 cases. Four out of the 28 patients presented the criteria for tumour rupture during surgery. Our patient presented both clinical and radiological features of tumour rupture. These were abdominal pain, decreased haemoglobin level, the presence of retroperitoneal fluid, and an irregular outline of the tumour capsule without maintaining periorgan fat reserves.

Brisse et al. retrospectively analysed 250 patients treated with the protocols recommended by SIOP [9]. Radiologic criteria set for retroperitoneal rupture included haemorrhage inside the tumour, retroperitoneal effusion, subcapsular and perirenal space haemorrhage and/or retroperitoneal tumour nodules separated from the primary tumour. Criteria of intraperitoneal rupture included intraperitoneal acute hemorrhage, intraperitoneal tumour nodules, tumour damage communicating with peritoneal effusion and/or visibility of mesenteric infiltration. In 57 patients clinical and radiological signs of pre-operative tumour rupture were described. Clinical criteria, such as abdominal pain, decrease in haemoglobin level, history of abdominal trauma were found in 39 children. The radiological features without clinical signs were observed in the next 18 patients. In 48 children with the radiological signs of retroperitoneal bleeding, the histopathological examination confirmed rupture only in 22 cases; these were all cases with intraperitoneal haemorrhage in CT examination. In our child apart from the previously listed features lymph node enlargement distant from the tumour was observed. The cystic part of the tumour described in CT might be the result of bleeding inside the tumour.

Khanna et al. performed a retrospective study, and they analysed CT examination from 204 research centres done in the years 2006 to 2010 [10]. Seventy children with Wilms tumour rupture were matched to 70 cases of uncomplicated nephroblastoma. The radiological features of preoperative tumour rupture included poorly circumscribed mass, perinephric fat stranding, peritumoural fat planes obscured, retroperitoneal fluid, ascites beyond the cul-de-sac, peritoneal implants, ipsilateral pleural effusion, and haemorrhage inside the tumour. This study confirmed relatively low sensitivity of CT in the detection of preoperative Wilms tumour rupture. Ascites beyond the cul-de-sac was found to be the most predictive of tumour rupture. Other highly predictive factors were fat stranding around the tumour and the presence of retroperitoneal fluid. In our patient fat stranding around the tumour and the presence of retroperitoneal fluid were observed.

Fukuzawa et al. indicated that large tumour mass and a high ratio of the tumour area to the abdominal area in a preoperative single horizontal CT (T/A ratio- the ratio of the tumour area to the abdominal area in a preoperative single horizontal computed tomography slice) are important risk factors for rupture during surgery [11]. The authors compared two groups of children with Wilms tumour. The T/A ratio in the ruptured group was significantly higher than in the non-ruptured group. This was an important factor also in our child.

Considering all clinical and radiological features of tumour rupture, the number of such cases will be very high. However, according to the SIOP report, preoperative tumour rupture is estimated at only $3 \%$ of all Wilms tumour cases $[12,13]$. Our patient did not manifest the features of acute abdomen and increasing anemisation caused by bleeding, despite big cystic lesions present in the tumour. So, neoadjuvant chemotherapy was the first line of treatment, followed by surgery.

All clinical and radiological symptoms might suggest pre-operative tumour rupture.

A decision about surgical treatment was made, despite the lack of clear reduction in tumour size after initial chemotherapy.

\section{CONCLUSIONS}

In summary, according to the data presented, it is very important to treat each patient individually. Not every child who meets the radiological criteria for preoperative tumour rupture should be upgraded. Features of preoperative tumour rupture in CT are often not clearly objec- 
tive. Perhaps in the future this will be possible thanks to the increased precision and more advanced equipment. Until then, all known features of tumour rupture must be precisely analysed to avoid overstaging and over-treatment. There is no doubt, however, that the radiological features of preoperative tumour rupture should be known to both radiologists and surgeons.

\section{DISCLOSURE}

The authors declare no conflict of interest.

\section{REFERENCES}

1. Brok J, Treger TD, Gooskens SL, et al. Biology and treatment of renal tumours in childhood. Eur J Cancer 2016; 68: 179-195.

2. Le Rouzic MA, Mansuy L, Galloy MA, et al. Agreement between clinicoradiological signs at diagnosis and radiohistological analysis after neoadjuvant chemotherapy of suspected Wilms tumor rupture: Consequences on therapeutic choices. Pediatr Blood Cancer 2019; e27674.

3. Graf N, Tournade MF, de Kraker J. The role of preoperative chemotherapy in the management of Wilms' tumor. The SIOP studies. International Society of Pediatric Oncology. Urol Clin North Am 2000; 27: 443-454.

4. Apoznański W, Patkowski D, Polok M, et al. Preoperative Wilms tumor rupture: Controversial diagnosis. Case report. Pediatr Pol 2017; 92: 786-788.

5. Shamberger RC, Guthrie KA, Ritchey ML, et al. Surgery-related factors and local recurrence of Wilms tumor in National Wilms Tumor Study 4. Ann Surg 1999; 229: 292- 297.

6. Burgers JM, Tournade MF, Bey P, et al. Abdominal recurrences in Wilms' tumours: a report from the SIOP Wilms' tumour trials and studies. Radiother Oncol 1986; 5: 175-182.

7. Vujanić GM, Sandstedt B, Harms D, et al. Revised International Society of Paediatric Oncology (SIOP) working classification of renal tumors of childhood. Med Pediatr Oncol 2002; 38: 79-82.

8. Ehrlich PF, Ritchey ML, Hamilton TE, et al. Quality assessment for Wilms' tumor: a report from the National Wilms' Tumor Study-5. J Pediatr Surg 2005; 40: 208- 213.

9. Brisse HJ, Schleiermacher G, Sarnacki S, et al. Preoperative Wilms tumor rupture: a retrospective study of 57 patients. Cancer 2008; 113: 202-213.

10. Khanna G, Naranjo A, Hoffer F, et al. Detection of Preoperative Wilms Tumor Rupture With CT: A Report From the Children's Oncology Group. Radiology 2013; 266: 610-617.

11. Fukuzawa H, Shiima $Y$, Mishima $Y$, et al. Predictive factor for intraoperative tumor rupture of Wilms tumor. Pediatr Surg Int 2017; 33: 91-95.

12. Godziński J, Weirich A, Tournade MF, et al. Primary nephrectomy for emergency: a rare event in the International Society of Paediatric Oncology Nephroblastoma Trial and Study no. 9. Eur J Pediatr Surg 2001; 11: 36-39.

13. Leape LL, Breslow NE, Bishop HC. The surgical treatment of Wilms' tumor: results of the National Wilms' Tumor Study. Ann Surg 1978; 187: 351-356. 